\title{
Evolutionary, Neural, and Statistical Approaches to Interval Clustering for Web Mining
}

\author{
Pawan Lingras, Rui Yan and Mofreh Hogo' \\ Department of Math and Computing Science, Saint Mary's University \\ Halifax, Nova Scotia, Canada, B3H 3C3; 'Department of Computer Science \\ and Engineering, Faculty of Electrical Engineering, Czech Technical \\ University, Karlovo Nam. 13, 12135 Prague 2, Czech Republic
}

\begin{abstract}
Rough set theory has been used extensively for supervised learning. Clustering within the context of rough set theory is attracting increasing interest among researchers. Typical clustering operations in data mining involve finding natural groupings of resources or users. Conventional clusters have crisp boundaries, i.e. each object belongs to only one cluster. The clusters and associations in data mining do not necessarily have crisp boundaries. An object may belong to more than one cluster. This paper describes three different methodologies based on properties of rough sets for developing interval representations of clusters. The first approach is based on Genetic Algorithms, the second approach is an adaptation of the K-means algorithm, and the last is based on Kohonen self-organizing maps. The paper also provides an experiment to illustrate the rough set based clustering of web users.
\end{abstract}

\section{KEYWORDS}

clustering, rough sets, Kohonen self-organizing maps, K-means, evolutionary computing

Reprint requests to: Pawan Lingras, Dept. of Math and Computing Science, Saint Mary's University, Halifax, Nova Scotia, Canada, B3H 3C3; e-mail: Pawan.Lingras@ stmarys.ca 


\section{INTRODUCTION}

Clustering in data mining faces several additional challenges compared with conventional clustering applications (Joshi \& Krishnapuram, 1998). The clusters tend to have fuzzy boundaries. Instead of an object precisely belonging to a cluster, it can be assigned a deg ree of fuzzy membership to one or more clusters. The likelihood is that an object might be a candidate for more than one cluster. Joshi and Krishnapuram (1998) argued that clustering operation in web mining involves modeling an unknown number of overlapping sets. The authors proposed the use of fuzzy clustering for grouping web resources. Similar arguments can be extended to clustering in data mining in general.

Rough set theory can provide another representation for overlapping clusters. Rough sets (Pawlak, 1984) enable us to represent such clusters using upper and lower bounds. Lingras (2001) described how a rough set theoretic clustering scheme can be represented using a rough set genome. In subsequent publications (Lingras \& West, 2002, Lingras et al., 2004), a modification of the K-means approach and the Kohonen neural network were proposed to create intervals of clusters based on rough set theory. All the three approaches have been used successfully in the clustering of web users. The K-means based and neural network approaches have been used for the clustering of supermarket customers as well. This paper provides a comprehensive summary of the rough set theoretic unsupervised learning based on evolutionary, neural, and statistical approaches. The results of rough set-based clustering of web users are also described as an illustration.

\section{ROUGH SET THEORY}

The notion of rough set was proposed by Pawlak (1982). This section provides a brief summary of the concepts from rough set theory.

Let $U$ denote the universe (a finite ordinary set), and let $R \subseteq U \times U$ be an equivalence (indiscernibility) relation on $U$. The pair $A=(U, R)$ is called an approximation space. 
The equivalence relation $R$ partitions the set $U$ into disjoint subsets. Such a partition of the universe is denoted by $U / R=\left\{E_{1}, E_{2}, . ., E_{n}\right\}$, where $E_{i}$ is an equivalence class of $R$. If two elements $u, v \in U$ belong to the same equivalence class $E \subset U / R$, we say that $u$ and $v$ are indistinguishable. The equivalence classes of $R$ are called the elementary or atomic sets in the approximation space $A=(U, R)$. The union of one or more elementary sets is called a composed set in $A$. The empty set $\varnothing$ is also considered a special composed set. $\operatorname{Com}(A)$ denotes the family of all composed sets. Because differentiating the elements within the same equivalence class is not possible, one might not be able to obtain a precise representation for an arbitrary set $X \subset U$ in terms of elementary sets in $A$. Instead, its lower and upper bounds may represent the set $X$. The lower bound $\underline{A}(X)$ is the union of all the elementary sets, which are subsets of $X$. The upper bound $\bar{A}(X)$ is the union of all the elementary sets that have a non-empty intersection with $X$.

The pair $(\underline{A}(X), \bar{A}(X))$ is the representation of an ordinary set of $\mathrm{X}$ in the approximation space $A=(U, R)$, or simply the rough set of $X$. The elements in the lower bound of $X$ definitely belong to $X$, while elements in the upper bound of $X$ may, or may not belong to $X$.

It can be verified that for any subsets, $X, Y \subset U$, the following properties hold (Pawlak, 1982):

$$
\begin{aligned}
& \underline{A}(X \cap Y)=\underline{A}(X) \cap \underline{A}(Y) \\
& \underline{A}(X \cup Y) \supseteq \underline{A}(X) \cup \underline{A}(Y) \\
& \bar{A}(X \cap Y) \subseteq \bar{A}(X) \cap \bar{A}(Y) \\
& \bar{A}(X \cup Y)=\bar{A}(X) \cup \bar{A}(Y) \\
& \bar{A}(-X)=-\underline{A}(X), \underline{A}(-X)=-\bar{A}(X) \\
& X \supseteq Y \Rightarrow(\underline{A}(X) \supset \underline{A}(Y), \bar{A}(X) \supseteq \bar{A}(Y)) \\
& \underline{A}(U)=\bar{A}(U)=U \\
& \underline{A}(\varnothing)=\bar{A}(\varnothing)=\varnothing
\end{aligned}
$$

Rough sets were proposed using equivalence relations. It is possible, however, to define a pair of upper and lower bounds $(\underline{A}(X), \bar{A}(X))$ or a 
Evolutionary, Neural, and Statistical Approaches to Interval Clustering

rough set for every set $X \subseteq U$, as long as the properties specified by Pawlak $(1982,1984)$ are satisfied. Yao et al. (1994) described various generalizations of rough sets by relaxing the assumptions of an underlying equivalence relation. Such a trend toward generalization is also evident in rough mereology proposed by Polkowski and Skowron (1996) and the use of information granules in distributed environment by Skowron and Stepaniuk (1999). The present study uses such a generalized view of rough sets. If one adopts a more restrictive view of rough set theory, the rough sets developed in this paper might have to be looked upon as interval sets.

Let us consider a hypothetical clustering scheme:

$$
U / P=\left\{X_{1}, X_{2}, . ., X_{k}\right\}
$$

that partitions the set $U$ based on certain criteria based on an equivalence relation $P$. The actual values of $X_{1}$ are not known. The clustering of web users is an example of such a hypothetical clustering scheme. A set of visitors can be classified into three classes depending on the predominant usage: studious, crammers, and workers. However, the actual sets corresponding to each one of these classes are not known. Let us assume that due to insufficient knowledge, precisely describing the sets $X_{i}, 1 \leq i<k$ in the partition is not possible. It is possible, however, to define each set using its lower and upper bounds based on the available information. In this study, the available information consists of web-access logs. Because the objects and clusters in the K-means algorithm are represented by vectors, we will use vector representations, $\mathbf{v}$ for an object and $\mathbf{x}_{i}$ for cluster $X_{i}$.

We are considering the upper and lower bounds of only a few subsets of $U$. Therefore, verifying all the properties of the rough sets is not possible (Pawlak, 1982, 1984). The family of upper and lower bounds of $\mathbf{x}_{1}$ are required, however, to follow some of the basic rough set properties such as:

An object $\mathbf{v}$ can be part of at most one lower bound

$$
\mathbf{v} \in \underline{A}\left(\mathbf{x}_{i}\right) \Rightarrow \mathbf{v} \in \bar{A}\left(\mathbf{x}_{i}\right)
$$

An object $\mathbf{v}$ is not part of any lower bound

$$
\Uparrow
$$

$\mathbf{v}$ belongs to two or more upper bounds. 
Property $(\mathrm{Cl})$ emphasizes that a lower bound is included in a set. If two sets are mutually exclusive, then their lower bounds should not overlap. Property $(\mathrm{C} 2)$ confirms that a lower bound is contained in the upper bound. Property (C3) is applicable to the objects in the boundary regions, which are defined as the differences between upper and lower bounds. The exact membership of objects in the boundary region is ambiguous. Therefore, property (C3) states that an object cannot belong to only a single boundary region. No te that $(\mathrm{C} 1)-(\mathrm{C} 3)$ are not necessarily independent or complete. Enumerating them, however, will be helpful in understanding the rough set adaptation of evolutionary, neural, and statistical clustering methods.

\section{GENETIC ALGORITHMS}

The origin of Genetic Algorithms (GAs) is attributed to Holland's (1975) work on cellular automata. Significant interest in GAs has been expressed over the last two decades. The range of applications of GAs includes such diverse areas as $\mathrm{j}$ ob shop scheduling, training neural nets, image feature extraction, and image feature identification (Buckles \& Petry, 1994). This section contains some of the basic concepts of genetic algorithms as described in Buckles and Petry (1994).

A genetic algorithm is a search process that follows the principles of evolution through natural selection. The domain knowledge is represented using a candidate solution called an organism. Typically, an organism is a single genome represented as a vector of length $n$ :

$$
c=\left(c_{i} \mid 1<i<n\right),
$$

where $c_{i}$ is called a gene.

A group of organisms is called a population. Successive populations are called generations. A generational GA starts from initial generation $G(0)$, and for each generation, $G(t)$ generates a new generation $G(t+1)$ using genetic operators such as mutation and crossover. The mutation operator creates new genomes by changing values of one or more genes at random. The crossover operator joins segments of two or more genomes to generate a new genome. 


\section{ROUGH SET GENOME AND ITS EVALUATION}

A rough set genome represents the entire clustering scheme. Let us assume that the objects are represented by $m$-dimensional vectors. The objective is to assign these $n$ objects to $k$ clusters.

Let $U=\left\{\mathbf{u}_{1}, \mathbf{u}_{2}, \cdots, \mathbf{u}_{n}\right\}$ be the set of objects that are to be partitioned into $k$ clusters given by: $U / P=\left\{\mathbf{x}_{1}, \mathbf{x}_{2}, \cdots, \mathbf{x}_{k}\right\}$.

The rough set genome will consist of $n$ genes, one gene per object. The gene for an object will be a string of bits that describes to which lower and upper approximations the object belongs. Properties (C1)-(C3) provide certain restrictions on the memberships. An object $u \in U$ can belong to the lower approximation of at most one cluster $\mathbf{x}_{i}$. If an object belongs to lower approximation of $\mathbf{x}_{l}$, then it also belongs to the upper approximation of $\mathbf{x}_{l}$. If an object does not belong to lower approximation of any $x_{i}$, then it belongs to upper approximation of at least two (possibly more) $\mathbf{x}_{\boldsymbol{l}}$.

Based on these observations, the string for a gene can be partitioned into two parts, lower and upper. Both the lower and upper parts of the string consist of $k$ bits each. The $i^{\text {th }}$ bit in lower/upper string tells whether the object is in the lower/upper approximation of $\mathbf{x}_{l}$.

If $\mathbf{u} \in \underline{A}\left(\mathbf{x}_{l}\right)$, then based on property (C2), $\mathbf{u} \in \bar{A}\left(\mathbf{x}_{l}\right)$. Therefore, the $i^{\text {th }}$ bit in both the lower and upper strings will be turned on. Based on property (C1), all the other bits must be turned off.

If $u$ is not in any of the lower approximations, then according to property (C3), it must be in two or more upper approximations of $\mathbf{x}_{l}, 1 \leq i \leq k$, and the corresponding $i^{\text {th }}$ bits in the upper string will be turned on.

Figure 1 shows examples of all the valid and some of the invalid genes for $k=3$. Genes gene, to gene $_{7}$ are all the acceptable values of genes for $k=$ 3. An object represented by gene ${ }_{1}$ belongs to $\bar{A}\left(\mathbf{x}_{1}\right)$ and $\bar{A}\left(\mathbf{x}_{2}\right)$. An object represented by gene ${ }_{6}$ belongs to $A\left(\mathbf{x}_{2}\right)$, and by property (C2) to $\bar{A}\left(\mathrm{x}_{2}\right)$.

Any other value not given by gene, to gene, is not valid. Figure 1 also shows four of the 57 invalid values. The invalidGene, is invalid because an object cannot be in $\underline{A}\left(\mathbf{x}_{1}\right)$ and not be in $\bar{A}\left(\mathbf{x}_{1}\right)$. The invalidGene, is invalid because an object cannot be in $\underline{A}\left(\mathbf{x}_{2}\right)$ and in $\bar{A}\left(\mathbf{x}_{3}\right)$. The invalidGene 3 is invalid because an object cannot be in $A\left(\mathbf{x}_{1}\right)$ and in $A\left(\mathbf{x}_{3}\right)$ at the same time. 
Valid genes

Lower Upper

\begin{tabular}{|l|l|l|l|l|l|l|}
\hline & $A\left(x_{3}\right)$ & $A\left(x_{1}\right)$ & $A\left(x_{1}\right)$ & $\bar{A}\left(x_{3}\right)$ & $\bar{A}\left(x_{2}\right)$ & $\bar{A}\left(x_{1}\right)$ \\
\hline gene1 & 0 & 0 & 0 & 0 & 1 & 1 \\
\hline gene2 & 0 & 0 & 0 & 1 & 0 & 1 \\
\hline gene3 & 0 & 0 & 0 & 1 & 1 & 0 \\
\hline gene4 & 0 & 0 & 0 & 1 & 1 & 1 \\
\hline gene5 & 0 & 0 & 1 & 0 & 0 & 1 \\
\hline gene6 & 0 & 1 & 0 & 0 & 1 & 0 \\
\hline gene7 & 1 & 0 & 0 & 1 & 0 & 0 \\
\hline
\end{tabular}

Some examples of invalid genes

\begin{tabular}{|l|l|l|l|l|l|l|}
\hline & $A\left(x_{3}\right)$ & $A\left(x_{2}\right)$ & $\mathcal{A}\left(x_{1}\right)$ & $\AA\left(x_{3}\right)$ & $\AA\left(x_{2}\right)$ & $\bar{\AA}\left(x_{1}\right)$ \\
\hline $\begin{array}{l}\text { invalid } \\
\text { gene1 }\end{array}$ & 0 & 0 & 1 & 0 & 0 & 0 \\
\hline $\begin{array}{l}\text { Invalid } \\
\text { gene2 }\end{array}$ & 0 & 1 & 0 & 1 & 1 & 0 \\
\hline $\begin{array}{l}\text { invalid } \\
\text { gene:3 }\end{array}$ & 1 & 0 & 1 & 0 & 0 & 0 \\
\hline $\begin{array}{l}\text { invalid } \\
\text { gene4 }\end{array}$ & 0 & 0 & 0 & 0 & 0 & 1 \\
\hline
\end{tabular}

Fig. 1: Genes in a rough set genome

Since the object represented by invalidGene, only belongs to $\bar{A}\left(\mathbf{x}_{1}\right)$, according to property $(C 3)$, it is invalid.

A genetic algorithm package such as the one used in the study (Wall, 1993) makes possible the description of a set of valid gene values or alleles. All the standard genetic operations will then only create genomes that have these values. Therefore, the conventional genetic operations can be used with rough set genomes in such a package.

The quality of a conventional clustering scheme is determined using the within-group-error (Sharma \& Werner, 1981) given by:

$$
\Delta=\sum_{i=1}^{k} \sum_{\mathbf{u}, \mathbf{v} \in \mathbf{x}_{\mathbf{l}}} d(\mathbf{u}, \mathbf{v}),
$$

where $u$ and $\mathbf{v}$ are objects from the same cluster $\mathbf{x}_{\boldsymbol{l}}$. 
The function $d$ provides the distance between two objects. The distance $d(\mathbf{u}, \mathbf{v})$ is given by:

$$
d(\mathbf{u}, \mathbf{v})=\sqrt{\frac{\sum_{j=1}^{m}\left(u_{j}-v_{\jmath}\right)^{2}}{m}}
$$

For a rough set clustering scheme, the exact values of clusters $x_{l}$ are not known. Given two objects $\mathbf{u}, \mathbf{v} \in U$ we have three distinct possibilities:

1. Both $\mathbf{u}$ and $\mathbf{v}$ are in the same lower approximation $A\left(\mathbf{x}_{l}\right)$.

2. Object $\mathbf{u}$ is in a lower approximation $A\left(\mathbf{x}_{i}\right)$ and $\mathbf{v}$ is in the corresponding upper approximation $\bar{A}\left(\mathbf{x}_{l}\right)$, and case 1 is not applicable.

3. Both $\mathbf{u}$ and $\mathbf{v}$ are in the same upper approximation $\bar{A}\left(\mathbf{x}_{1}\right)$, and cases 1 and 2 are not applicable.

For these possibilities, one can define three corresponding types of within-group-errors, $\Delta_{1}, \Delta_{2}$, and $\Delta_{3}$ as:

$$
\begin{aligned}
& \Delta_{1}=\sum_{i=1}^{k} \sum_{\mathbf{u}, \mathbf{v} \in \underline{A}\left(\mathbf{x}_{1}\right)} d(\mathbf{u}, \mathbf{v}) \\
& \left.\Delta_{2}=\sum_{i=1}^{k} \sum_{\mathbf{u} \in \underline{A}\left(\mathbf{x}_{i}\right), \mathbf{v} \in A\left(\mathbf{x}_{1}\right), \mathbf{v} \notin \underline{A}\left(\mathbf{x}_{i}\right)} \mathbf{u}, \mathbf{v}\right) \\
& \left.\Delta_{3}=\sum_{i=1}^{k} \sum_{\mathbf{u}, \mathbf{v} \in \bar{A}\left(\mathbf{x}_{i}\right), \mathbf{u}, \mathbf{v} \notin \underline{A}\left(\mathbf{x}_{i}\right)} u, \mathbf{u}\right)
\end{aligned}
$$

The total error of rough set clustering will then be a weighted sum of these errors:

$$
\Delta_{\text {total }}=w_{1} \times \Delta_{1}+w_{2} \times \Delta_{2}+w_{3} \times \Delta_{3}
$$

Since $\Delta_{!}$corresponds to situations where both objects definitely belong to the same cluster, the weight $w_{1}$ should have the highest value. On the other hand, $\Delta_{3}$ corresponds to the situation where both objects may or may not 
belong to the same cluster. Hence, $w_{3}$ should have the lowest value. In other words, $w_{1}>w_{2}>w_{3}$. There are many possible ways of developing an error measure for rough set clustering. The measure $\Delta_{\text {total }}$ is perhaps one of the simplest. More sophisticated alternatives can be used, depending upon the application.

If we used genetic algorithms to minimize $\Delta t o t a l$, then the genetic algorithms would try to classify all the objects in upper approximations by taking advantage of the fact that $w_{1}>w_{3}$. This may not necessarily be the best clustering scheme. We want the rough set clustering to be as precise as possible. Therefore, a precision measure needs to be used in conjunction with $\Delta$ total for evaluating the quality of a rough set genome. A possible precision measure can be defined in the following (Pawlak, 1982) as:

$$
\text { precision }=\frac{\text { (Number of objectsclassifiedin lower approximaions) }}{\text { (Total number of objects) }}
$$

The objective of the genetic algorithms will then be to maximize the quantity:

$$
\text { objective }=p \times \text { precision }+\frac{e}{\Delta_{\text {total }}}
$$

where $p$ and $e$ are additional parameters. The parameter $p$ describes the importance of the precision measure in determining the quality of a rough set genome. Higher values of $p$ will result in smaller boundary region. Similarly, $e$ indicates the importance of within-group errors relative to the size of boundary region. Perhaps we should note here that adding five new parameters $w_{1}, w_{2}, w_{3}, p$, and $e$ was necessary for the rough set theoretic evolutionary clustering. The values of these parameters need to be adjusted based on the application, similar to other genetic algorithm parameters.

\section{K-MEANS ALGORITHM}

K-means clustering is one of the most popular statistical clustering techniques (Hartigan \& Wong, 1979; MacQueen, 1967). The name K-means 
originates from the means of the $k$ clusters that are created from $n$ objects. Let us assume that the objects are represented by $m$-dimensional vectors. The objective is to assign these $n$ objects to $k$ clusters. Each cluster is also represented by an $m$-dimensional vector, which is the centroid or mean vector for that cluster. The process begins by randomly choosing $k$ objects as the centroids of the $k$ clusters. The objects are assigned to one of the $k$ clusters based on the minimum value of the distance $d\left(\mathbf{v}, \mathbf{x}_{i}\right)$ between the object vector $\mathbf{v}$ and the cluster vector $\mathbf{x}_{i}$.

After the assignment of all the objects to various clusters, the new centroid vectors of the clusters are cal culated as:

$$
\mathbf{x}_{i j}=\frac{\sum_{\mathbf{v} \in \mathbf{v}_{i}} v_{j}}{\text { Size of cluster } \mathbf{x}_{\boldsymbol{I}}}, 1 \leq J \leq m
$$

The process stops when the centroids of clusters stabilize, i.e. the centroid vectors from the previous iteration are identical to those generated in the current iteration.

\section{ADAPTATION OF K-MEANS TO ROUGH SET THEORY}

Incorporating rough sets into $\mathrm{K}$-means clustering requires the addition of the concept of lower and upper bounds. Equation 8, which used to calculate the centroids of clusters, has to be modified to include the effects of lower as well as upper bounds. The modified centroid calculations for rough sets are then given by:

if $\underline{A}\left(\mathbf{x}_{i}\right) \neq \varnothing$ and $\bar{A}\left(\mathbf{x}_{i}\right)-\underline{A}\left(\mathbf{x}_{l}\right) \neq \varnothing$,

$$
\mathbf{x}_{i i}=\frac{\sum_{\mathbf{v} \in A\left(\mathbf{x}_{i}\right)} v_{j}}{\left|\underline{A}\left(\mathbf{x}_{i}\right)\right|}, 1<j \leq m
$$

else if $\underline{A}\left(\mathbf{x}_{l}\right)=\varnothing$ and $\bar{A}\left(\mathbf{x}_{i}\right)-\underline{A}\left(\mathbf{x}_{i}\right) \neq \varnothing$, 


$$
\mathbf{x}_{\text {ij }}=\frac{\sum_{\mathbf{v} \in\left(\widetilde{A}\left(\mathbf{x}_{i}\right)-\underline{A}\left(\mathbf{x}_{i}\right)\right)} v_{j}}{\left|\bar{A}\left(\mathbf{x}_{l}\right)-\underline{A}\left(\mathbf{x}_{i}\right)\right|}, 1 \leq j \leq m
$$

else

$$
\mathbf{x}_{i j}=w_{l o w e r} \times \frac{\sum_{\mathbf{v} \in \underline{A}\left(\mathbf{x}_{i}\right)}}{\left|\underline{A}\left(\mathbf{x}_{i}\right)\right|}+w_{\text {upper }} \times \frac{\sum_{\mathbf{v} \in\left(\bar{A}\left(\mathbf{x}_{i}\right)-A\left(\mathbf{x}_{i}\right)\right)} v_{j}}{\left|\bar{A}\left(\mathbf{x}_{i}\right)-\underline{A}\left(\mathbf{x}_{i}\right)\right|}, 1 \leq j \leq m
$$

The parameters $w_{\text {lower }}$ and $w_{\text {upper }}$ correspond to the relative importance of lower and upper bounds, and $w_{\text {lower }}+w_{\text {upper }}=1$. It can be easily seen that above formulation is a generalization of Eq. 8. If the upper bound of each cluster is equal to its lower bound, then the clusters will be conventional clusters. Therefore, the boundary region $\bar{A}(x)-A(x)$ will be empty, and the second term in the equation will be ignored.

The next step in the modification of the K-means algorithms for rough sets is to design criteria to determine whether an object belongs to the upper or to the lower bound of a cluster, given as follows.

Let $d\left(v, x_{i}\right)=\min _{1 \leq j \leq k} d\left(v, x_{j}\right)$. The ratios $\frac{d(v \cdot x)}{d\left(v, x_{j}\right)}, 1 \leq j \leq k$, are used Let $T=\left\{\mathrm{j}: \mathrm{d}\left(\mathbf{v}, \mathbf{x}_{\mathrm{i}}\right) / \mathrm{d}\left(\mathbf{v}, \mathbf{x}_{\mathrm{j}}\right)<\right.$ threshold,$\left.i \neq j\right\}$.

If $T \neq \varnothing, \mathrm{v} \in \bar{A}\left(\mathbf{x}_{l}\right)$ and $\mathbf{v} \in \bar{A}\left(\mathbf{x}_{j}\right), \forall j \in T$.

Furthermore, $\mathbf{v}$ is not part of any lower bound. The above criterion guarantees that property (C3) is satisfied. Otherwise, if $T=\varnothing, \mathbf{v} \in \underline{A}\left(\mathbf{x}_{l}\right)$ such that $\mathrm{d}\left(\mathbf{v}, \mathbf{x}_{\mathbf{i}}\right)$ is the minimum for $1<i<k$. In addition, by property (C2), $v \in \bar{A}\left(\mathbf{x}_{i}\right)$.

We should emphasize that the approximation space $\mathbf{A}$ is not defined based on any predefined relation on the set of objects. The upper and lower bounds are constructed based on the criteria described above.

The rough K-means algorithm, described above, depends on three parameters $w_{\text {lower }}, w_{\text {upper, }}$, and threshold. Experimentation with various values of the parameters is necessary to develop a reasonable rough set clustering. 


\section{KOHONEN SELF-ORGANIZING MAPS}

Figure 2 illustrates the conventional Kohonen network architecture for the one-dimensional case. The unsupervised learning with the Kohonen rule (1988) uses the competitive learning approach. In competitive learning, the output neurons compete with each other. The winner output neuron has the output of 1 , the rest of the output neurons have outputs of 0 . The competitive learning is suitable for classifying a given pattern into exactly one of the mutually exclusive clusters. The network is used to group patterns represented by $m$-dimensional vectors into $k$ groups. The network consists of two layers. The first layer is called the input layer and the second layer is called the Kohonen layer. The network receives the input vector for a given pattern. If the pattern belongs to the $i^{\text {th }}$ group, then the $t^{\text {th }}$ neuron in the Kohonen layer has an output value of one, and the other Kohonen layer neurons have output values of zero. Each connection is assigned a weight $w_{i j}$. The weights of all the connections to a Kohonen layer neuron make up an $m$-dimensional weight vector $\mathbf{w}_{i}$. The weight vector $\mathbf{w}_{i}$ for a Kohonen layer neuron is the vector representation of the group corresponding to that neuron. For any input vector $\mathbf{v}$, the network compares the input with the weight vector for a group using the measure such as Eq. (4).

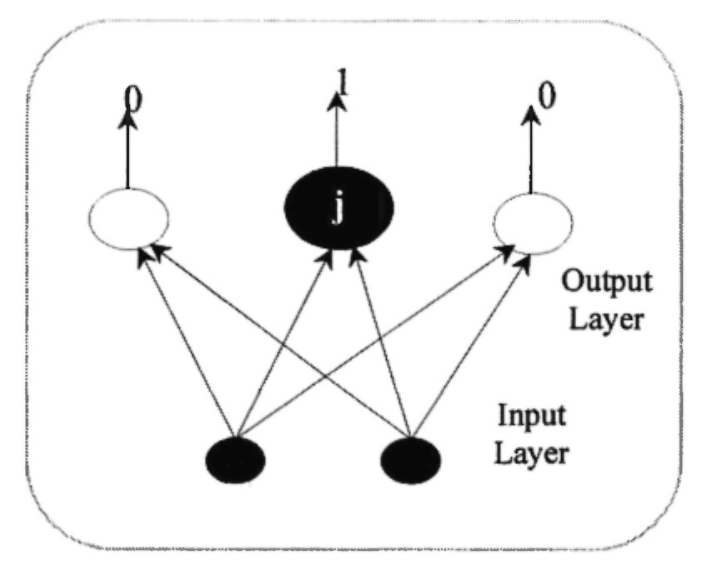

Fig. 2: Kohonen neural network 
The pattern $\mathbf{v}$ belongs to the group with minimum value for $d\left(\mathbf{w}_{i}, \mathbf{v}\right)$. The Kohonen neural network generates the clusters through a learning process as follows: Initially, the network connections are assigned somewhat arbitrary weights. The training set of input vectors is presented to the network several times. For each iteration, the weight vector $\mathbf{w}_{i}$ for a group that is closest to the pattern $\mathbf{v}$ is modified using the equation:

$$
\mathbf{w}_{i}^{\text {new }}=\mathbf{w}_{i}^{\text {old }}+\alpha(t) \times\left(\mathbf{v}-\mathbf{w}_{i}^{\text {old }}\right),
$$

where $\alpha(t)$ is a learning factor starting with a high value at the beginning of the training process and is gradually reduced as a function of time.

\section{MODIFICATIONS OF KOHONEN ALGORITHM}

The rough set-based Kohonen algorithm uses the concept of lower and upper bounds in the equations for updating the weights of the winners. The Kohonen rough set architecture is similar to the conventional Kohonen architecture, consisting of two layers- - an input layer and the Kohonen rough set layer (rough set output layer). The two layers are fully connected. Each input layer neuron has a feed forward connection to each output layer neuron. Figure 3 illustrates the Kohonen rough set neural network architecture for one-dimensional case. A neuron in the Kohonen layer consists of two parts, a lower neuron and an upper neuron. The lower neuron has an output of 1 , if an object belongs to the lower bound of the cluster. Similarly, a membership in the upper bound of the cluster will result in an output of 1 from the upper neuron. Because an object belonging to the lower bound of a cluster also belongs to its upper bound, when lower neuron has an output of 1 , the upper neuron also has an output of 1 . Membership in the upper bound of a cluster, however, does not necessarily imply the membership in its lower bound. Therefore, the upper neuron contains the lower neuron. Figure 3 provides several cases to explains outputs from the Kohonen rough set neural network works based on properties (C1), (C2), and (C3). Figure 3(a-c) shows some of the possible outputs, whereas Figure $3(d-f)$ shows some of the invalid outputs from the network. Figure 3(a) shows a case where an object belongs to lower 
bound of cluster $x_{2}$. Based on the property (C2), the object also belongs to the upper bound of $x_{2}$. Figure 3(b) shows a situation where an object belongs to the upper bounds of clusters $x_{1}$ and $x_{2}$. The object in Fig. 3(c) belongs to the upper bounds of clusters $x_{1}, x_{2}$, and $x_{3}$. Figure $3(d)$ shows an invalid situation where an object belongs only to the upper bound of the cluster $x_{3}$, which is a violation of the property (C3). Figure 3(e) shows a violation of property (C1), where an object belongs to lower bound of $x_{3}$ as well as the upper bound of $\mathbf{x}_{2}$. Similarly, a violation of property (C2) can be seen in an invalid case shown in Fig. 3(f). Here the object belongs only to the lower bound of cluster $\mathbf{x}_{3}$ and not to its upper bound. The modification of the Kohonen algorithm must ensure that the properties (C1)-(C3) are obeyed by avoiding cases such as the ones shown in Fig. 3(d-f).

Interval clustering provides good results, if initial weights are obtained by running the conventional Kohonen learning.

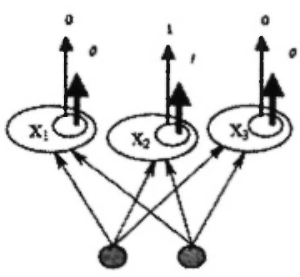

(a)

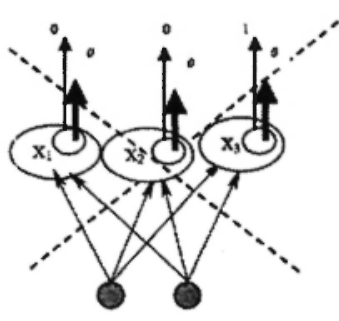

(d)

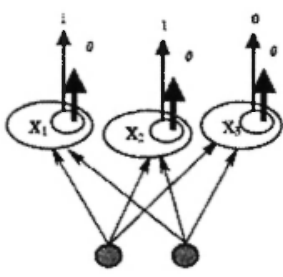

(b)

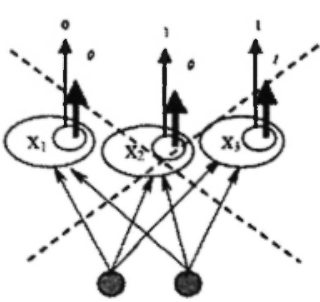

(c)

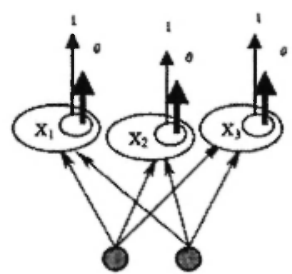

(c)

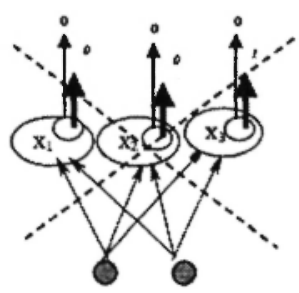

(f)

Fig. 3: Modified Kohonen neural network 
Step 1: Initialize weights from $m$ inputs to the $k$ output nodes using the conventional Kohonen algorithm.

Step 2: For each object vector, $\mathbf{v}$, let $\mathrm{d}\left(\mathbf{v}, \mathbf{x}_{\mathrm{i}}\right)$ be the distance between itself and the weight vector $\mathbf{x}_{i}$ of cluster $X_{i}$.

Step 3: The next step in the modification of the Kohonen algorithm for obtaining rough sets is to design criteria to determine whether an object belongs to the upper or lower bounds of a cluster.

Let $d\left(v, x_{i}\right)=\min _{1 \leq j \leq k} d\left(v, x_{j}\right)$.

The ratios $\frac{d\left(v, x_{i}\right)}{d\left(v, x_{j}\right)}, 1 \leq j \leq k$,

are used to determine the membership of $\mathbf{v}$ as follows.

Let $T=\left\{\mathrm{j}: \mathrm{d}\left(\mathbf{v}, \mathbf{x}_{\mathbf{j}}\right) / \mathrm{d}\left(\mathbf{v}, \mathbf{x}_{\mathrm{j}}\right)<\right.$ threshold, $\left.i \neq j\right\}$.

If $T \neq \varnothing, \mathbf{v} \in \bar{A}\left(\mathbf{x}_{l}\right)$ and $\mathbf{v} \in \bar{A}\left(\mathbf{x}_{j}\right), \forall j \in T$.

Furthermore, $\mathbf{v}$ is not part of any lower bound. The above criterion guarantees that property (C3) is satisfied. The weight vectors only $\mathbf{x}_{i}$ and $\mathbf{x}$, are modified as:

$$
\begin{aligned}
& \mathbf{x}_{i}^{\text {new }}=\mathbf{x}_{i}^{\text {old }}+\alpha_{\text {upper }}(t) \times\left(\mathbf{v}-\mathbf{x}_{i}^{\text {old }}\right) \\
& \mathbf{x}_{j}^{\text {new }}=\mathbf{x}_{j}^{\text {old }}+\alpha_{\text {upper }}(t) \times\left(\mathbf{v}-\mathbf{x}_{j}^{\text {old }}\right) .
\end{aligned}
$$

Otherwise, if $T=\varnothing, \mathbf{v} \in \underline{A}\left(\mathbf{x}_{1}\right)$ such that $\mathrm{d}\left(\mathbf{v}, \mathbf{x}_{\mathrm{i}}\right)$ is the minimum for $1<i<k$. In addition, by property (C2), $\mathbf{v} \in \bar{A}\left(\mathbf{x}_{1}\right)$. The weight vector $\mathbf{x}_{\mathrm{i}}$ is modified as:

$$
\mathbf{x}_{i}^{\text {new }}=\mathbf{x}_{i}^{\text {old }}+\alpha_{\text {lower }}(t) \times\left(\mathbf{v}-\mathbf{x}_{i}^{\text {old }}\right)
$$

Usually, $\alpha_{\text {lower }}(t)>\alpha_{\text {upper }}(t)$.

Step 4: If the clustering remains unchanged from the previous iteration, stop. Otherwise, go to Step 2, as long as maximum number of iterations is not reached.

It can be easily verified that the above algorithm preserves properties (C1)-(C3). We should emphasize that the approximation space $A$ is not 
defined based on any predefined relation on the set of objects. The upper and lower bounds are constructed based on the criteria described above. Lingras et al. (2004) conducted experiments with web logs on three web sites, suggesting that the modifications of the Kohonen neural networks provide reasonable interval set representations of clusters.

\section{EXPERIMENTAL EVALUATION}

This section reports the results of rough set clustering using genetic algorithms. Because of space restrictions, results from other methods cannot be included. The study data were obtained from the web-access logs of the introductory first year course in computing science at Saint Mary's University. The initial number of students in the course was 180 . The number decreased over the course of the semester to approximately 130 to 140 . The students in the course come from a wide variety of backgrounds, such as computing science major hopefuls, students taking the course as a required science course, and students taking the course as a science or general elective. As is common in a first year course, students' attitudes towards the course also vary a great deal. It was hoped that the profile of visits would reflect some of the distinctions among the students. For the initial analysis, we assumed that the visitors could fall into one of the following three categories:

1. Studious: These visitors download the current set of notes. Because they have downloaded a limited/current set of notes, they probably study classnotes on a regular basis.

2. Crammers: These visitors download a large set of notes. This indicates that they have stayed away from the classnotes for a long period of time. They are planning for pre-test cramming.

3. Workers: These visitors are mostly working on class or lab assignments or accessing the discussion board.

The rough set clustering scheme was expected to specify lower and upper bounds for these classes.

It was hoped that the above mentioned variety of user behaviors would be identifiable based on the number of web accesses, types of documents 
downloaded, and the time of day. Certain areas of the website were protected, and users could access them only by using their IDs and passwords. The activities in the restricted parts of the website consisted of submitting a user profile, changing a password, submission of assignments, viewing the submissions, accessing the discussion board, and viewing current class marks. The rest of the website was public. The public portion consisted of viewing course information, lab manual, classnotes, class assignments, and lab assignments.

If the users only accessed the public website, then their IDs would be unknown. Therefore, the web users were identified based on their IP addresses, which also ensured that the user privacy was protected. A visit from an IP address started when first request was made from the IP address. The visit continued as long as the consecutive requests from the IP address had sufficiently small delay.

The web logs were preprocessed to create an appropriate representation of each user corresponding to a visit. The abstract representation of a web user is a critical step that requires a good knowledge of the application domain. Previous personal experience with the students in the course suggested that some students download preliminary notes before a class and an updated copy after the class. Some students view the notes online only on a regular basis. Some students download the notes around important days such as midterm and final examinations. In addition, many accesses occur on Tuesdays and Thursdays, when the in-laboratory assignments are due. Onand off-campus points of access can also provide some indication of a user's objectives for the visit. Based on some of these observations, we decided to use the following attributes for representing each visitor:

1. On campus/off campus access.

2. Day time/Night time access: 8 a.m. to 8 p.m. was considered day time.

3. Access during lab days or non-lab days: All labs and classes were held on Tuesday and Thursday. The visitors on these days are more likely to be workers.

4. Number of hits.

5. Number of classnotes downloads. 


\begin{tabular}{|l|r|r|r|r|r|}
\hline Cluster & $V_{1}$ & $V_{2}$ & $V_{3}$ & $V_{4}$ & $V_{5}$ \\
\hline$\underline{A}($ Studious $)$ & 0.67 & 0.71 & 0.43 & 24 & 5 \\
$\bar{A}($ Studious $)$ & 0.69 & 0.74 & 0.45 & 28 & 8 \\
A $($ Crammer $)$ & 0.58 & 0.69 & 0.38 & 26 & 8 \\
$\overline{\bar{A}}($ Crammer $)$ & 0.67 & 0.75 & 0.45 & 29 & 9 \\
$\underline{A}$ (Worker $)$ & 0.68 & 0.81 & 0.45 & 19 & 3 \\
$\bar{A}($ Worker $)$ & 0.68 & 0.78 & 0.44 & 20 & 4 \\
\hline
\end{tabular}

Fig. 4: Vector representation of clusters

The first three attributes had binary values of 0 or 1 . The last two values were normalized. Because the classnotes were the focus of the clustering, the last variable was assigned higher importance. For the experiments reported in this paper, the data were restricted to 2 weeks around the midterm examination. These 2 weeks logged 54528 entries. The data preparation identified 3243 visits. The visitors that did not download any notes clearly fall in the worker category. Therefore, the clustering was restricted to those 1264 visits that downloaded at least one classnotes file.

The rough set-based genetic algorithm was used to obtain the intervals of three clusters. Each gene was allowed to take the valid values shown in Fig. 1. Each rough set genome corresponds to a rough set clustering scheme. Genetic algorithms attempt to evolve a genome such that the value of an objective function is maximal. The objective function given by Eq. (7) was used during the evolution process. Because the precision is in the range $[0,1]$ and the total error $\Delta$ total can be as high as $6 \times 10^{7}$, we decided to modify the parameters $e$ as:

$$
e=e^{\prime} \times \Delta_{\max }
$$

Various values of $e^{\prime}$ and $p$, as well as other genetic parameters like the number of generations, probabilities of crossover and mutation, and population sizes were used.

Genetic algorithms were run for several generations, ranging from 100 to 1000. In Eq. (5), $w_{l}=1.0, w_{2}=0.5, w_{3}=0.25$ were set. The value of $\Delta_{\max }$ 
was found to be approximately equal to $6 \times 10^{7}$. Different pairs of $\left(p, e^{\prime}\right)$ tried included $(0.5,0.5),(0.6,0.4),(0.7,0.3),(0.8,0.2)$, and $(0.9,0.1)$. The probability of crossover was 0.9 , and the mutation rate was 0.01 . Based on subjective evaluation, the most appropriate clustering scheme was observed for 150 generations, population size of 100 , and $(p, e)=(0.7,0.3)$.

Figure 4 shows the average values of the five variables used in the clustering. It was possible to classify the three clusters as Studious, Crammers, and Workers. The Workers had the lowest number of hits per visit (19 to 20). Workers downloaded only 3 to 4 documents, which probably corresponded to the sample programs from the lab manuals. The crammers, on the other hand, had the highest number of hits per visit (26 to 29). Crammers downloaded 8 to 9 notes per visit. The studious visitors had wider variations. Their average hits were between 24 and 28 , and average notes downloads were between 5 and 8 . Because of the higher weights associated with the hits and classnotes, the rest of the attributes seemed to have similar values for all the categories. The crammers, however, tended to have a higher tendency to access the site from off-campus locations. Crammers also visited the website at night more frequently than the other groups. The cardinalities of the upper and lower bounds of clusters are shown in Fig. 5.

\begin{tabular}{|l|c|}
\hline Cluster & Size \\
\hline$\underline{\bar{A}}($ Studious $)$ & 132 \\
$\bar{A}($ Studious $)$ & 511 \\
$\underline{\bar{A}}($ Crammer $)$ & 112 \\
$\bar{A}($ Crammer $)$ & 477 \\
$\underline{\bar{A}}($ Worker $)$ & 364 \\
$\bar{A}($ Worker $)$ & 651 \\
\hline
\end{tabular}

Fig. 5: Cardinalities of resulting upper and lower bounds 
It is also interesting to note that the ranges of lower and upper bounds for studious and crammers overlap. This seems to suggest that the proposed clustering satisfies one of the important criteria of overlapping cluster boundaries (Joshi \& Krishnapuram, 1998).

To verify that some visitors belonged to multiple categories, an additional analysis was also carried out by taking intersections of all the upper bounds. The intersections provide some indication of the visitors who could not be precisely classified-263 visitors were identified as either studious or crammers; 185 visitors could be classified as either workers or studious; and 171 visitors could have been either crammers or workers. Finally, 69 visitors were in all the three upper bounds.

Whereas the GAs based approach was applicable to 1264 web visitors, it could not be applied to a large number of web visitors because of the high computational requirements. Both K-means and Kohonen-based rough clustering were applicable to clustering more than 8000 web visitors. These approaches were also useful for clustering more than 100,000 clusters. The results obtained by all three methods were comparable. The Kohonen approach, however, was significantly faster than the Gas-based approach. The $\mathrm{K}$-means-based approach was slightly faster than the Kohonen-based rough set clustering. The adaptive nature of the Kohonen algorithm, however, can be advantageous in certain applications.

Lingras et al. (2003) also applied the fuzzy C-means algorithm for the web-user data set described in this paper. The fuzzy $\mathrm{C}$-means can give a more detailed degree of memberships than those given by lower and upper bounds. Although such detailed memberships can be useful in some applications, they can be difficult to analyze. The rough set representation of clusters provides a compromise between conventional crisp clusters and detailed fuzzy memberships.

\section{SUMMARY AND CONCLUSIONS}

The clusters in data mining do not necessarily have precise boundaries. Rough sets can provide representation of such clusters. This paper has 
described modifications of Genetic Algorithms-based clustering, the K-means algorithm, and Kohonen self-organizing maps based on the concept of lower and upper bounds. All three approaches have been successfully used in realworld applications. The paper described an application of rough set based clustering of web users. Both K-means and Kohonen approaches required significantly smaller computational time. The $\mathrm{K}$-means approach is slightly more efficient than the Kohonen algorithm.

\section{ACKNOWLEDGMENT}

The authors would like to thank the Natural Sciences and Engineering Research Council of Canada, the Faculty of Graduate Studies and Research, Saint Mary's University, and the Government of Egypt for their financial support.

\section{REFERENCES}

Buckles, B.P., and Petry F.E. 1994. Genetic algorithms, Los Alamitos, California, USA, IEEE Computer Press.

Hartigan, J.A., and Wong M.A. 1979. Algorithm AS136: A K-means clustering algorithm, Applied Statistics, 28, 100-108.

Holland, J.H. 1975. Adaptation in natural and artificial systems, Ann Arbor, Michigan, USA, University of Michigan Press.

Joshi A., and Krishnapuram R. 1998. Robust fuzzy clustering methods to support web mining, Proceedings of the workshop on data mining and knowledge discovery, SIGMOD '98, Seattle (1998) 15/1-15/8 .

Lingras P. 2001. Unsupervised rough set classification using GAs, Journal of Intelligent Information Systems, 16, 215-228.

Lingras P. 2002. Rough set clustering for web mining, Proceedings of 2002 IEEE international conference on fuzzy systems.

Lingras P., and West C. 2004. Interval set clustering of web users with rough $\mathrm{K}$-means, Journal of Intelligent Information Systems, 23, 5-16.

Lingras, P., Hogo, M., and Snorek M. 2004. Interval set clustering of web users using modified Kohonen self-organizing maps based on the properties of rough sets, Web Intelligence and Agent Systems, 2, 217-225.

Lingras, P., Yan, R., and West, C. 2003. Fuzzy C-means clustering of web users for educational sites, in Advances in artificial intelligence, Lecture notes in computer science, 2671, Lecture notes in artificial intelligence, 

Approaches to Interval Clustering

edited by Xiang, Y., and Brahim, C., Berlin, Germany-New York, New York, NY, USA, Springer, 557-562.

MacQueen, J. 1967. Some methods for classification and analysis of multivariate observations, in: Proceedings of fifth Berkeley symposium on mathematical statistics and probability, edited by Le Cam L.M., and Neyman, J., 1, 281-297.

Pawlak Z. 1984. Rough classification, International Journal of Man-Machine Studies, 20, 469-483.

Pawlak Z. 1982. Rough Sets, International Journal of Information and Computer Sciences, 11, 145-172.

Pawlak Z. 1992. Rough sets: theoretical aspects of reasoning about data, Dordrecht, The Netherlands, Kluwer Academic Publishers.

Polkowski L., and Skowron A. 1996. Rough mereology: A new paradigm for approximate reasoning, International Journal of Approximate Reasoning, $15,333-365$.

Sharma S.C., and Werner A. 1981. Improved method of grouping provincewide permanent traffic counters, Transportation Research Record, 815, Transportation Research Board, Washington DC, USA, 13-18.

Skowron A., and Stepaniuk J. 1999. Information granules in distributed environment, in: New directions in rough sets, data mining, and granular-soft computing, Lecture notes in computer science, 1711, Lecture notes in artificial intelligence, edited by Zhong, N. Skowron, A., and Ohsuga, S., Berlin, Germany, Springer-Verlag, 357-365.

Kohonen T. 1988. Self-organization and associative memory, Berlin, Germany, Springer Verlag.

Wall M. 1993. Galib: A C++ Library of Genetic Components, available online at http://lancet.mit.edu/ga/.

Yao, Y.Y., Li, X., Lin, T.Y., and Liu Q. 1994. Representation and classification of rough set models, Proceedings of third international workshop on rough sets and soft computing, San Jose, California, 630637. 